Paños Castro, J. (2017). Educación emprendedora y metodologías activas para su fomento. Revista Electrónica Interuniversitaria de Formación del Profesorado, 20 (3), 33-48. DOI: http://dx.doi.org/10.6018/reifop.20.3.272221

\title{
Educación emprendedora y metodologías activas para su fomento
}

\author{
Jessica Paños Castro \\ Universidad de Deusto
}

\section{Resumen}

El emprendimiento no solo se refiere a la creación de nuevas empresas y/o negocios. Hoy en día a su vez es entendida como una competencia que engloba un conjunto de habilidades y destrezas como son la creatividad, el liderazgo, el trabajo en equipo, la innovación, la toma de decisiones...; todas ellas demandadas en el ámbito personal, social y profesional. Las instituciones educativas deberían promover está competencia al verse contemplada en las leyes educativas (Ley 2/2006; Ley 8/2013) y planes de estudios universitarios. Ahora bien, ¿qué metodologías se deberían emplear para desarrollar está competencia?, ¿En qué consisten? y ¿qué competencias fomenta cada una de ellas? El objetivo de este artículo es responder a estos interrogantes realizando una revisión bibliográfica de los últimos artículos publicados, pero antes se hace un recorrido por el término emprendimiento para a continuación señalar las habilidades y destrezas que engloba esta competencia, y el objetivo de la educación emprendedora.

\section{Palabras clave}

Emprendimiento; educación emprendedora; competencias genéricas; métodos de enseñanza.

\section{Entrepreneurship education and active methodologies for its promotion}

\begin{abstract}
Entrepreneurship not only refers to the creation of new companies and/or businesses. Today it is also understood as a competence that includes a set of skills and abilities such as creativity, leadership, teamwork, innovation, decision making...; all of them demanded in personal, social and professional level. Educational institutions should promote this competence as it is introduced in education laws (Law 2/2006; Law 8/2013) and in university
\end{abstract}


curricula. Now, what methods should be used to develop this competence? What does it consist on? And, what competencies encourage each of them? The aim of this article is to answer these questions by carrying out a literature review of the latest articles published, but first it is a tour around the term entrepreneurship; then, the skills and abilities that include this competence are pointed out and finally, the objective of entrepreneurship education.

\section{Key words}

Entrepreneurship; entrepreneurship education; generic competences; teaching methods.

\section{Introducción}

El emprendimiento hoy en día está de moda (Garavan y O'Cinneide, 1994; Arruti, 2016) no solo en el ámbito económico, social y político, sino también en la educación. Así lo reflejan las últimas leyes educativas promulgadas en España como son la Ley Orgánica para la mejora de la Calidad Educativa (Ley 8/2013) y la Ley Orgánica de Educación (Ley 2/2006). No obstante, conviene señalar que anteriormente otras leyes educativas ya se hacían eco del emprendimiento. Así, en España la Ley Orgánica de Calidad de la Educación (Ley 10/2002) incorporó por primera vez la iniciativa emprendedora indicando que "el espíritu emprendedor es necesario para hacer frente a la evolución de las demandas de empleo en el futuro" (Ley 10/2002, 2002, p. 45189). En el año 2013, la ley 14/2013 de apoyo a los emprendedores y a su internacionalización subrayaba que "el personal docente que imparte las enseñanzas que integran el sistema educativo deberá adquirir las competencias y habilidades relativas al emprendimiento" (Ley 14/2013, de 27 de septiembre, de apoyo a los emprendedores y a su internacionalización, 2013, p.78800).

Algo similar ha ocurrido en los demás países de la Unión Europea donde poco a poco se ha ido incorporando en los currículos educativos aunque con una mayor presencia en los años 2009-2010 (Comisión Europea, 2016b). De hecho, el emprendimiento no es un concepto novedoso pero quizás, en estos tiempos de crisis económica, política, social y educativa se hace indispensable generar un valor transformador desde las aulas (Jiménez, Elías y Silva, 2014). Es preciso señalar que la generación más joven del siglo XXI es la generación más emprendedora desde la revolución industrial (Kuratko, 2005). Según Volkmann (2004), el estudio del emprendimiento, especialmente en Europa, es parcialmente joven aunque desde comienzos del siglo XXI se está convirtiendo en una fuerte disciplina académica.

Volkmann (2004) subraya que las metodologías son un factor decisivo para el éxito de las universidades emprendedoras pero a día de hoy escasean las investigaciones (Winslow, Solomon y Tarabishy, 1999). Es por ello que la finalidad de este artículo es ofrecer un marco teórico que aporte luz a los educadores de todas las etapas educativas, a académicos y a otras partes interesadas a la hora de seleccionar las metodologías activas que mejor favorecen la competencia de emprender. No obstante, este artículo también puede ser interesante para el desarrollo de otras competencias y/o disciplinas dado que las metodologías expuestas al final del documento no son exclusivamente de la competencia emprendedora. Además, tal y como señala Fiet (2001b) es conveniente considerar la investigación sobre el emprendimiento y la enseñanza para tomar decisiones acordes. Resumiendo, este análisis presenta principalmente dos aportes a la literatura. Por un lado, proporcionar una revisión literaria, de reflexión y divulgación. Y por otro lado, ofrecer de manera sintética una descripción de las principales metodologías activas que fomentan la competencia emprendedora, relacionándolas con las habilidades y destrezas que se trabajan. 
La metodología de este estudio es de carácter cualitativo basada en una revisión bibliográfica utilizando el metabuscador Océano, herramienta de búsqueda de recursos bibliográficos que incluye más de cien bases de datos de impacto tanto nacionales como internacionales. Los descriptores utilizados para la búsqueda han sido los siguientes: "emprendimiento metodologías", "emprendimiento métodos", "enseñanza emprendimiento", "entrepreneurship pedagogies", "entrepreneurship teaching approaches", "entrepreneurship pedagogical methods", "entrepreneurship methods" y "entrepreneurship methodologies". Después de realizar esta búsqueda, se llevó a cabo una revisión bibliográfica de los trabajos que recogían las metodologías activas que ayudan a potenciar la competencia de emprender. Previo al análisis de las metodologías, se presenta una pequeña contextualización del tema exponiendo qué es el emprendimiento, la competencia emprendedora y la importancia de ésta en la educación.

\section{¿Qué es emprender?}

La palabra emprendimiento proviene del francés entrepreneur que significa pionero. Según Fayolle, Gailly y Lassas-Clerc (2006), el emprendimiento tiene dos perspectivas. Por un lado, en términos de impacto directo que se refiere a la creación de nuevas empresas y/o empleo. $\mathrm{Y}$ por otro lado, en términos de impacto indirecto. Esto es, el estímulo del espíritu emprendedor. Estos mismos autores sostienen que el emprendimiento no está exclusivamente relacionado con la creación de nuevas empresas o negocios. Esta idea se ver reforzada por autores como Ruiz (2015), Kuratko (2005) y Kirby (2004) al afirmar que el emprendedor no necesariamente debe convertirse en empresario. Dicho en otras palabras, el término es mucho más amplio que el simple hecho de montar una empresa o negocio.

Damián (2013), por su parte, presenta un doble enfoque. Por un lado está el enfoque utilitarista que hace referencia a la acción de montar una empresa y/o negocio. El segundo enfoque es más bien social y amplio ya que se refiere al conjunto de competencias transversales que cualquier persona puede emplear a lo largo de su vida para ser ciudadano activo y obtener empleo pero no necesariamente para montar una empresa o negocio. Para la Comisión Europea (2008) el emprendimiento es la capacidad de transformar las ideas en acción. Esta se considera una competencia transversal y clave para todos los seres humanos, útil en todos los ámbitos de la vida, tanto personal, social como profesional (Comisión Europea, 2016a; Comisión Europea, 2008).

Resumiendo, el término emprender es un concepto amplio, polisémico y huidizo (Damián, 2013). Es más, es caótico, complejo y carece de cualquier noción de linealidad (Neck y Greene, 2011). Como se observa, actualmente no existe una definición estándar (Hoffmann, Fuglsang y Vestergaard, 2012; Kirby, 2004).

\section{El emprendimiento como competencia}

Una competencia es "la capacidad para resolver problemas en cualquier situación y, especialmente, cuando se trata de situaciones nuevas o diferentes a las ya conocidas, y en diversos contextos de actuación" (Zabala y Arnau, 2014, p.9).

Así, el emprendimiento es considerado una competencia. Ahora bien, la amplitud de la competencia ha hecho que se formule de distintas maneras. La Ley Orgánica para la Mejora de la Calidad Educativa (Ley 8/2013) la denomina "sentido de iniciativa y espíritu emprendedor", la Ley Orgánica de Educación (Ley 2/2006) "autonomía e iniciativa personal", la Organización para la Cooperación y el Desarrollo Económicos "actuar autónomamente" y la Unión Europea, por el contrario, "espíritu emprendedor" (Ruiz, 2015). No solo la formulación varía sino también la tipología. En el año 2002 el Consejo 
Europeo de Lisboa de la Unión Europea y la Ley Orgánica de Educación (Ley 2/2006) la incluyeron como competencia básica, el Parlamento Europeo y la Ley Orgánica para la Mejora de la Calidad Educativa (Ley 8/2013), en cambio, la incorporaron como competencia clave, la Unión Europea como competencia transversal y el proyecto Tuning (González y Wagenaar, 2003) como competencia genérica sistémica.

Una persona emprendedora posee un conjunto de habilidades, cualidades y comportamientos. Como se detalla más adelante, actualmente existen discrepancias en los elementos distintivos del emprendimiento como competencia (Comisión Europea, 2016a).

Siguiendo a Gibb y Hannon (2007) se requieren las siguientes destrezas y habilidades: búsqueda de oportunidades, iniciativa, compromiso, locus of control (hace referencia a la valoración positiva de que los hechos dependan de uno mismo (Villa y Poblete, 2007)), capacidad para trabajar en red, pensamiento estratégico, capacidad de negociación, capacidad de persuasión, orientación al logro, creatividad y asunción de riesgos. Según Villa y Poblete (2007), la competencia "espíritu emprendedor" es una competencia sistémica que se relaciona con el liderazgo, la innovación, la creatividad, la adaptación al entorno, la automotivación, la toma de decisiones, la iniciativa y la visión de futuro. Kirby (2004), por su parte, lo asocia con la creatividad, la necesidad de logro, el locus of control, la autonomía, la intuición y la asunción de riesgos.

En una reciente investigación se recogen las áreas competenciales sobre la competencia emprendedora. Se trata del marco EntreComp (Comisión Europea, 2016a) que abarca 3 áreas competenciales ("ideas y oportunidades", "recursos" y "acción") integrando diferentes competencias, como se aprecia en la tabla 1.

Tabla 1.

Marco EntreComp. Relación áreas competenciales

\begin{tabular}{ll}
\hline Áreas & Competencias \\
\hline Ideas y oportunidades & $\begin{array}{l}\text { Búsqueda de oportunidades } \\
\text { Creatividad } \\
\text { Visión } \\
\text { Valorar ideas } \\
\text { Pensamiento ético y sostenible }\end{array}$ \\
Recursos & Mutoeficacia y conciencia de uno mismo \\
& Motivación y perseverancia \\
& Educación financiera y económica \\
& Movilizar a otros \\
Acción & Tener iniciativa \\
& Planificación y gestión \\
& Hacer frente a incertidumbres, riesgos y ambigüedades \\
& Trabajar con otros \\
& Aprendizaje mediante la experiencia \\
\hline
\end{tabular}




\section{La educación emprendedora}

Hasta hace poco tiempo se pensaba que el emprendimiento o ciertas dimensiones del mismo no se podían enseñar (Kuratko, 2005). Dicho en otras palabras, que los emprendedores nacen y no se hacen ya que es una cuestión de personalidad y una característica psicológica. Actualmente, esta cuestión ya está resuelta dado que autores como Volkmann (2004), Kuratko (2003, 2005), la Comisión Europea (2016a), Hindle (2007), y Henry, Hill y Leitch (2005) afirman que las competencias emprendedoras se pueden enseñar y aprender en diferentes lugares y modos. Si no fuera así, ¿cuál es el objetivo de la educación emprendedora? (Powell, 2013). Además, tal y como afirma Kuratko "la cuestión de si el emprendimiento se puede enseñar o no es obsoleta" (Kuratko, 2002, p.8). Evidentemente ciertas habilidades se adquieren de manera no intencionada a través de las vivencias personales; como puede ocurrir con la competencia emprendedora. Ahora bien, la educación juega un papel fundamental para desarrollar la creatividad, el talento y la capacidad de innovación; características propias de la persona emprendedora (Asociación Red GEM España, 2016). Así, la educación emprendedora es definida como:

La disciplina que engloba los conocimientos y habilidades "sobre" o "con el fin de que" el emprendimiento en general sea reconocido como parte de los programas educativos correspondientes a las enseñanzas primaria, secundaria o terciaria (superior) en las instituciones educativas oficiales de cualquier país. (Coduras, Levie, Kelley, Saemundsson y Schott, 2010, p.13).

Con todo ello, el profesorado juega un papel fundamental en todas las etapas educativas, desde las edades más tempranas hasta la educación superior: "los profesores y profesoras pasan a convertirse en el recurso crítico más valioso de nuestra sociedad, pues deben ser los actores que deben protagonizar el cambio" (De la Fuente, Vera y Cardelle-Elawar, 2012, p.958). De hecho, recientemente se viene hablando del término teacherpreneur que es una combinación de profesor y emprendedor. Los teacherpreneurs son profesores con pasión, seguros, flexibles, que rompen las reglas preestablecidas y cuentan con expertos en sus clases (Arruti, 2013).

Es preciso señalar que la universidad hoy en día se ha sumado a la tercera misión, conocido también como modelo de triple hélice (universidad-industria-gobierno). En otras palabras, además de la enseñanza y la investigación, el nuevo papel de la universidad es contribuir a la economía (Etzkowitz y Leydesdorff, 1998). Es más, el profesorado universitario debería promover la formación emprendedora pero lamentablemente no ocurre así en muchos casos: "El profesorado universitario tiende a primar la formación específica de cada disciplina en detrimento de la formación emprendedora, entre otros motivos por una falta de desconocimiento por su parte de cómo enseñar su disciplina de manera emprendedora" (Ripollés, 2011, p.84). Una razón de peso podría ser porque en la literatura escasean los buenos consejos (Hindle, 2007). Así, en el presente artículo, como se apuntaba en la introducción, se pretende orientar a los docentes, académicos y otras partes interesadas en la educación en esta cuestión.

\section{Metodologías activas para el desarrollo de la competencia de emprender}

Una metodología es:

Un conjunto de decisiones sobre los procedimientos a emprender y sobre los recursos a utilizar en las diferentes fases de un plan de acción que, organizados y secuenciados coherentemente con los objetivos pretendidos 
en cada uno de los momentos del proceso, nos permiten dar una respuesta a la finalidad última de la tarea educativa. (De Miguel, 2005, p.36).

La mayoría de los autores clasifican las metodologías en dos grandes grupos. Por un lado, están los métodos tradicionales, también conocidos como métodos pasivos. $\mathrm{Y}$ por otro lado, los métodos innovadores, activos o basados en la acción (Samwel, 2010).

Una de las tareas de los docentes es saber programar y elegir las metodologías más adecuadas para alcanzar una determinada competencia (Rosales, 2013; De Miguel, 2006). Es decir, las metodologías son el vehículo para desarrollar las competencias (Fernández, 2006).

Es preciso redefinir las metodologías de enseñanza (Unión Europea, 2012) dado que el empleo de las metodologías tradicionales, unidireccionales y pasivas para trabajar las competencias están obsoletas (De la Fuente, Vera y Cardelle-Elawar, 2012; Comisión Europea, 2008; Honig, 2004; Neck y Greene, 2011). Esto es, es necesario un cambio metodológico para extraer el máximo rendimiento a la competencia emprendedora (Pellicer, Álvarez y Torrejón, 2013; Bager, 2011). Por consiguiente, debemos usar metodologías activas donde el alumno adopte un papel activo, participativo (Ripollés, 2011; Bager, 2011) y se sitúe en el centro del proceso de enseñanza-aprendizaje (Kirby, 2004), esto es, que sea él el protagonista y el profesor el guía, asesor y/o facilitador, de lo contrario los alumnos difícilmente podrán desarrollar las competencias emprendedoras (Comisión Europea, 2008; Garavan y O'Cinneide, 1994; Jones y Iredale, 2010; Zahra y Welter, 2008; Hytti y O'Gorman, 2004; Ripollés, 2011; Jiménez, Elías y Silva, 2014). Además, con el inicio del proceso de Bolonia, en 1999, el modelo basado en la enseñanza derivó al centrado en el aprendizaje (Álvarez, 2016). Lamentablemente aún hay una fuerte dependencia hacia el enfoque centrado en el profesor (Zahra y Welter, 2008) y al uso de metodologías tradicionales (Jones y Iredale, 2010).

Como se apuntaba en la introducción, la mayor parte de los países de la Unión Europea han incluido en sus currículums la educación emprendedora (Comisión Europea, 2016b). No obstante, en estos documentos no se suele detallar qué metodologías utilizar. La literatura tampoco ofrece directrices claras sobre la selección adecuada (Balan y Metcalfe, 2012). De hecho, a nivel estatal la Orden ECD/65/2015 en el anexo II, orientaciones para facilitar el desarrollo de estrategias metodológicas que permitan trabajar por competencias en el aula, únicamente señala que "las metodologías que mejor favorecen la participación activa, la experimentación y el aprendizaje funcional para el desarrollo de competencias son: el aprendizaje por proyectos, el estudio de casos, los centros de interés y el aprendizaje basado en problemas" (Orden ECD/65/2015, 2015, p.7003). A nivel autonómico sólo Castilla y León dispone de directrices específicas en relación a las estrategias metodológicas dirigidas a fomentar la cultura emprendedora. Así se indica en la resolución de 30 de agosto de 2013 en la instrucción dos, orientaciones pedagógicas, en el apartado b, metodología: "El trabajo por proyectos, el aprendizaje basado en problemas, el aprendizaje-servicio (ApS) y las estrategias de aprendizaje cooperativo" (Resolución de 30 de agosto de 2013 por la que se establecen orientaciones pedagógicas y se determinan las actuaciones dirigidas a fomentar la cultura emprendedora, 2013, p.62084). En esta misma orden se señala la importancia de la participación e implicación del alumno.

En los siguientes párrafos de este apartado se detallan los resultados del análisis bibliográfico realizado, esto es, las metodologías activas más usuales en los programas de emprendimiento. Como se presenta más adelante, existe una amplia variedad de metodologías aunque algunas son más efectivas y apropiadas que otras (Comisión Europea, 2008). Incluso la selección puede variar en función del país (Zahra y Welter, 2008). La 
selección de una u otra dependerá primeramente de los objetivos que queramos lograr (Balan y Metcalfe, 2012; Hytti y O'Gorman, 2004) y de la composición del grupo (Koch, 2003).

Fiet (2001a) apunta que es necesario enseñar teoría para que los alumnos sepan hacer predicciones y tomar decisiones consecuentes. Ahora bien, y como es lógico, este tipo de clases pueden ser aburridas. Ante ello, subraya que las clases no tienen que ser predecibles y que el papel del profesor es limitado. De acuerdo con la investigación de Winslow, Solomon y Tarabishy (1997), los métodos más empleados en las instituciones educativas fueron la creación de planes de negocios (87\%), el estudio de casos (78\%) y las visitas de ponentes (69\%). Hytti y O'Gorman (2004), por su parte, realizaron un análisis sobre las metodologías que se empleaban en 50 cursos sobre emprendimiento en Europa. Los resultados evidenciaron que los métodos más utilizados fueron las simulaciones, los juegos, los métodos tradicionales (lecciones magistrales), las competiciones, los workshops, las visitas, la creación de empresas y el entrenamiento práctico. Samwel (2010) asimismo realizó un trabajo de campo revisando 21 artículos. En el identificó 26 métodos distintos aunque los más frecuentes fueron los siguientes, como se refleja en la siguiente figura:

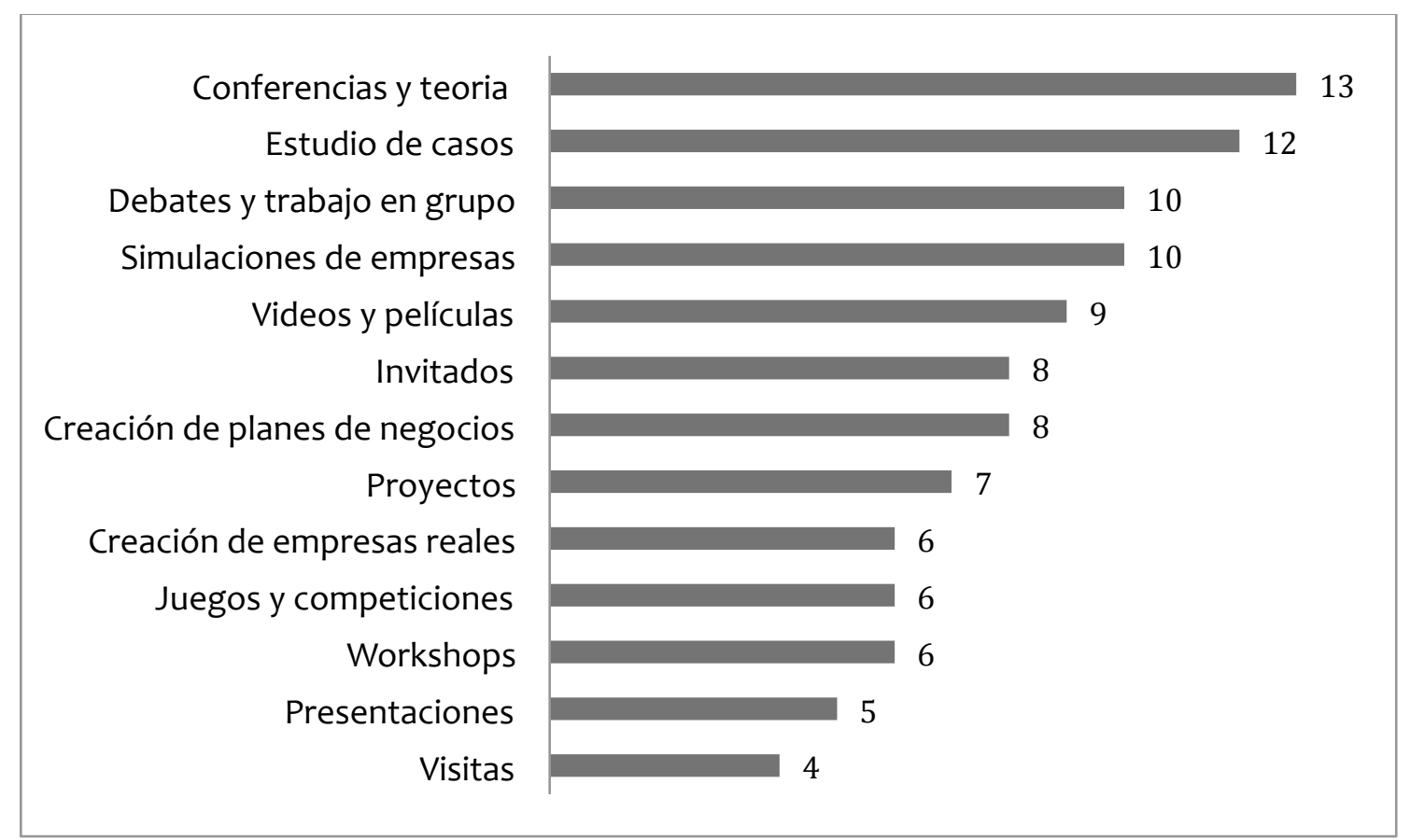

Figura 1. Número de artículos donde se recogen las metodologías empleadas en los cursos de emprendimiento.

En una reciente investigación realizada por Ruskovaara y Pihkala (2013) se recogen los siguientes datos en cuanto a los métodos más utilizados a partir de una muestra de 521 docentes: discusiones, relatos, presentaciones, entrevistas y escritos. Los juegos, por el contrario, tenían un menor uso. Estos mismos autores señalan que las visitas fuera del aula son más comunes que las visitas al centro. Asimismo, llama la atención la afirmación que plantean al indicar que no existe suficiente información disponible sobre la relación entre los métodos y los resultados obtenidos a través de la educación emprendedora.

A continuación se presenta la referencia a algunos autores que plantean algunas metodologías para el desarrollo de la competencia emprendedora: 
Tabla 2.

Relación de autores sobre las metodologías más adecuadas para estimular la competencia emprendedora.

\begin{tabular}{|c|c|}
\hline $\begin{array}{l}\text { Metodologías más adecuadas para } \\
\text { estimular la competencia } \\
\text { emprendedora }\end{array}$ & Relación de autores \\
\hline Estudio de casos & $\begin{array}{l}\text { Volkmann (2004) } \\
\text { Fayolle, Gailly y Lassas-Clerc (2006) } \\
\text { Zahra y Welter (2008) } \\
\text { Solomon, Duffy y Tarabishy (2002) } \\
\text { Jiménez (2015) } \\
\text { Koch (2003) }\end{array}$ \\
\hline $\begin{array}{l}\text { Juegos de negocios empresariales } \\
\text { y simulaciones }\end{array}$ & $\begin{array}{l}\text { Volkmann (2004) } \\
\text { Jiménez (2015) } \\
\text { Koch (2003) } \\
\text { Neck y Greene (2011) }\end{array}$ \\
\hline $\begin{array}{l}\text { Aprender haciendo (learning by doing) } \\
\text { en situaciones reales }\end{array}$ & $\begin{array}{l}\text { Fayolle, Gailly y Lassas-Clerc (2006) } \\
\text { Coduras et al. (2010) }\end{array}$ \\
\hline Aprendizaje experiencial & $\begin{array}{l}\text { Comisión Europea (2008) } \\
\text { Coduras et al. (2010) } \\
\text { Sherman, Sebora y Digman (2008) }\end{array}$ \\
\hline Charlas con emprendedores & $\begin{array}{l}\text { Fayolle, Gailly y Lassas-Clerc (2006) } \\
\text { Solomon, Duffy y Tarabishy (2002) }\end{array}$ \\
\hline $\begin{array}{l}\text { Aprendizaje basado en el diseño } \\
\text { (design-based learning) }\end{array}$ & Neck y Greene (2011) \\
\hline Creación de planes de negocios & $\begin{array}{l}\text { Solomon, Duffy y Tarabishy (2002) } \\
\text { Sherman, Sebora y Digman (2008) }\end{array}$ \\
\hline Aprendizaje por proyectos & $\begin{array}{l}\text { Jiménez (2015) } \\
\text { Koch (2003) } \\
\text { Pellicer, Álvarez y Torrejón (2013) }\end{array}$ \\
\hline Aprendizaje basado en problemas & $\begin{array}{l}\text { Jiménez (2015) } \\
\text { Pellicer, Álvarez y Torrejón (2013) }\end{array}$ \\
\hline Aprendizaje cooperativo & $\begin{array}{l}\text { Jiménez (2015) } \\
\text { Pellicer, Álvarez y Torrejón (2013) }\end{array}$ \\
\hline Aprendizaje y servicio & Pellicer, Álvarez y Torrejón (2013) \\
\hline
\end{tabular}


Según la Comisión Europea (2016b), las metodologías más comunes para el desarrollo de la competencia emprendedora suelen ser el aprendizaje activo, el aprendizaje basado en proyectos, el aprendizaje experiencial y las actividades fuera del aula. La Unión Europea (2012), sin embargo, considera que el estudio de casos, las simulaciones de negocios, el trabajo en grupo y los workshops son los métodos más efectivos. Mientras que el Ministerio de Educación, Cultura y Deporte (2016) indica que el aprendizaje y servicio solidario o el aprendizaje cooperativo cobran protagonismo en los programas de emprendimiento.

Resumiendo, existe una gran variedad de métodos de enseñanza-aprendizaje para trabajar la competencia emprendedora (Seikkula-Leino, Ruskovaara, Ikavalko, Mattila y Rytkola, 2010). Aunque los métodos tradicionales están profundamente arraigados (Fiet, 2001b) son preferibles las metodologías interactivas, orientadas a la acción y poco convencionales (Zahra y Welter, 2008; Winslow, Solomon y Tarabishy, 1997). Dicho en otras palabras, la competencia emprendedora no se puede enseñar eficazmente utilizando métodos tradicionales (Hoffmann, Fuglsang y Vestergaard, 2012) dado que los estudiantes son más pasivos y se les prepara para ser emprendedores (Arasti, Kiani y Imanipour, 2012). Sin embargo, hasta la fecha no hay suficientes investigaciones que describan el impacto o la efectividad de cada metodología para la enseñanza del emprendimiento (Winslow, Solomon y Tarabishy, 1999). De hecho, el emprendimiento al ser tan caótico y al tener diferentes definiciones, las metodologías también adoptan esta representación (Solomon, 2007).

Con el objetivo de evitar confusiones y de ofrecer orientaciones sobre las metodologías activas, en la siguiente tabla se muestran las principales metodologías que hacen hincapié en la competencia emprendedora, ofreciendo un referente teórico y un análisis de las principales competencias que desarrolla cada método y que guarda relación con la competencia emprendedora, como se ha recogido en el apartado tres.

Tabla 3.

Metodologías para el desarrollo de la competencia emprendedora

\begin{tabular}{|l|l|}
\hline Metodología & Descripción \\
\hline $\begin{array}{l}\text { Estudio } \\
\text { casos }\end{array}$ & $\begin{array}{l}\text { Metodología que consiste en analizar, gestionar, tomar decisiones y } \\
\text { buscar soluciones eficaces a problemáticas reales de actualidad o } \\
\text { simuladas (De Miguel, 2006; Jiménez, 2015; Labrador y Andreu, 2008; } \\
\text { Fernández, 2006; Zabala y Arnau, 2014) y que está abierta a distintas } \\
\text { interpretaciones (Coloma, Jiménez y Sáez, 2007). El objetivo es acercar } \\
\text { al alumno a su futura profesión (Labrador y Andreu, 2008). }\end{array}$ \\
\hline Competencias que se trabajan y que están relacionadas con la competencia emprendedora \\
\hline - Pensamiento crítico (Jiménez, 2015; Zabala y Arnau, 2014) \\
- Comunicación oral y escrita (Jiménez, 2015; De Miguel, 2006) \\
- $\quad$ Resolución de problemas (Jiménez, 2015; Zabala y Arnau, 2014) \\
- $\quad$ Planificación (Coloma, Jiménez y Sáez, 2007; De Miguel, 2006) \\
- Toma de decisiones (Coloma, Jiménez y Sáez, 2007; Zabala y Arnau, 2014)
\end{tabular}




\begin{tabular}{|c|c|}
\hline $\begin{array}{ll}\text { - } & \text { Visión de } \\
\text { - } & \text { Motivació } \\
\text { - } & \text { Iniciativa } \\
\text { - } & \text { Responsa } \\
\text { - } & \text { Autonom } \\
\text { - } & \text { Comunica }\end{array}$ & $\begin{array}{l}\text { futuro (Coloma, Jiménez y Sáez, 2007) } \\
\text { n para el logro (Coloma, Jiménez y Sáez, 2007) } \\
\text { De Miguel, 2006) } \\
\text { bilidad (De Miguel, 2006) } \\
\text { a (De Miguel, 2006) } \\
\text { ción interpersonal (Zabala y Arnau, 2014) }\end{array}$ \\
\hline $\begin{array}{l}\text { Simulaciones y } \\
\text { juegos }\end{array}$ & $\begin{array}{l}\text { Metodología que permite acercar al alumno a experiencias y desafíos } \\
\text { reales (Jiménez, 2015). }\end{array}$ \\
\hline $\begin{array}{ll}\text { - } & \text { Creativida } \\
\text { - } & \text { Iniciativa } \\
\text { - } & \text { Capacidac } \\
\text { - } & \text { Toma de } \\
\text { - } & \text { Autonom }\end{array}$ & $\begin{array}{l}\text { d (Jiménez, 2015) } \\
\text { Jiménez, 2015) } \\
\text { les interpersonales (Fernández, 2006) } \\
\text { decisiones (Zabala y Arnau, 2014) } \\
\text { a (Zabala y Arnau, 2014) }\end{array}$ \\
\hline $\begin{array}{l}\text { Aprendizaje } \\
\text { Basado en } \\
\text { Proyectos }\end{array}$ & $\begin{array}{l}\text { Metodología que consiste en diseñar, elaborar y realizar un proyecto con } \\
\text { el objetivo de resolver problemas auténticos o abordar una tarea (De } \\
\text { Miguel, 2006; Jiménez, 2015) }\end{array}$ \\
\hline $\begin{array}{lr}\text { - } & \text { Pensamie } \\
\text { - } & \text { Trabajo el } \\
\text { - } & \text { Comunica } \\
\text { - } & \text { Pensamie } \\
\text { - } & \text { Toma de } \\
\text { - } & \text { Iniciativa } \\
\text { - } & \text { Planificac } \\
\text { - } & \text { Responsa }\end{array}$ & $\begin{array}{l}\text { nto crítico (Jiménez, 2015) } \\
\text { equipo (Jiménez, 2015; De Miguel, 2006) } \\
\text { ción oral y escrita (Jiménez, 2015; De Miguel, 2006) } \\
\text { nto creativo (Fernández, 2006) } \\
\text { decisiones (De Miguel, 2006) } \\
\text { De Miguel, 2006) } \\
\text { ón (De Miguel, 2006) } \\
\text { bilidad (De Miguel, 2006) }\end{array}$ \\
\hline $\begin{array}{l}\text { Aprendizaje } \\
\text { cooperativo }\end{array}$ & $\begin{array}{l}\text { Trabajo en grupos reducidos (Jiménez, 2015) para lograr metas comunes } \\
\text { (Labrador y Andreu, 2008). No se debe confundir el aprendizaje } \\
\text { cooperativo con el trabajo en grupo. Este último suele tender a ser } \\
\text { competitivo (Coloma, Jiménez y Sáez, 2007). El aprendizaje cooperativo, } \\
\text { por el contrario, es en colaboración entre iguales (Coloma, Jiménez y } \\
\text { Sáez, 2007; Labrador y Andreu, 2008). Esto es, se da una estrategia de } \\
\text { corresponsabilidad para lograr metas grupales (De Miguel, 2006) dado } \\
\text { que uno alcanza sus objetivos si, y sólo si, los demás consiguen los suyos. } \\
\text { Gracias a la zona de desarrollo próximo el sujeto puede adquirir nuevos } \\
\text { contenidos, resolver problemas y realizar tareas (De Miguel, 2006). }\end{array}$ \\
\hline
\end{tabular}


- Comunicación oral y escrita (Jiménez, 2015; Labrador y Andreu, 2008; Fernández, 2006; De Miguel, 2006; García, Traver y Candela, 2001)

- Trabajo en equipo (Jiménez, 2015; Labrador y Andreu, 2008)

- Comunicación interpersonal (García, Traver y Candela, 2001): como son la asertividad (Jiménez, 2015), la empatía (Jiménez, 2015), la confianza y el apoyo mutuo (Labrador y Andreu, 2008)

- $\quad$ Espíritu de superación (Jiménez, 2015)

- Gestión del tiempo (Jiménez, 2015)

- Liderazgo (Labrador y Andreu, 2008)

- Toma de decisiones (Labrador y Andreu, 2008)

- Resolución de conflictos (Labrador y Andreu, 2008; De Miguel, 2006)

- Autoconfianza (Labrador y Andreu, 2008)

Aprendizaje

Basado

Problemas
En pequeños grupos detectar, identificar y resolver problemas (Jiménez, en 2015; De Miguel, 2006; Fernández, 2006; Labrador y Andreu, 2008; Zabala y Arnau, 2014) poniendo en práctica los conocimientos previos (Moust, Bouhuijs y Schmidt, 2007).

- Pensamiento crítico (Jiménez, 2015; Labrador y Andreu, 2008; Zabala y Arnau, 2014)

- Trabajo en equipo (Jiménez, 2015; De Miguel, 2006; Zabala y Arnau, 2014)

- Resolución de problemas (Jiménez, 2015; Moust, Bouhuijs y Schmidt, 2007; De Miguel, 2006; Zabala y Arnau, 2014)

- Autonomía (Moust, Bouhuijs y Schmidt, 2007)

- Comunicación interpersonal (Moust, Bouhuijs y Schmidt, 2007; Zabala y Arnau, 2014)

- Creatividad (Labrador y Andreu, 2008; Zabala y Arnau, 2014)

- Toma de decisiones (De Miguel, 2006; Zabala y Arnau, 2014)

- Comunicación oral y escrita (De Miguel, 2006; Zabala y Arnau, 2014)

\section{Discusión y conclusiones}

Actualmente, el emprendimiento está presente en diferentes contextos; no solo en el ámbito económico sino también en el ámbito social, personal y educativo. En lo referente a este último, en España por primera vez la Ley Orgánica de Calidad de la Educación (Ley 10/2002) incluyó el espíritu emprendedor en el sistema educativo, y posteriormente lo hizo la Ley Orgánica de Educación (Ley 2/2006), hasta la actual ley educativa, la Ley Orgánica para la Mejora de la Calidad Educativa (Ley 8/2013). Los demás países de la Unión Europea, más o menos, han seguido la misma dirección aunque a distinto ritmo.

Siendo las cosas así, los docentes hoy en día deberían integrar la competencia emprendedora en todas las etapas educativas, desde la educación básica obligatoria hasta la educación superior, esto es, deberían ser facilitadores de la cultura emprendedora. Es 
evidente que si queremos lograr una cultura emprendedora la formación en emprendimiento es necesaria. Así, las instituciones educativas no deberían quedarse pasivas y deberían adaptarse a las nuevas demandas del siglo XXI respondiendo ante los cambios. De esta manera, se ofrecería a los alumnos una educación de calidad, que les capacite para resolver problemas ante situaciones reales y así, desarrollen al máximo sus potencialidades.

Como resultado del artículo se deduce que no existe unanimidad sobre el término emprendimiento ni sobre las competencias que éste engloba, ni siquiera sobre qué métodos de enseñanza emplear. Lo que sí queda reflejado es que las metodologías pasivas, unidireccionales y centradas en el profesor no son eficaces per se. Además, ningún método por si solo es suficiente para dar respuesta a las competencias (Zabala y Arnau, 2014). Dependiendo de las competencias que se quieran trabajar se utilizara una u otra metodología, incluso más de una también (De Miguel, 2005).

Indiscutiblemente las metodologías son el vehículo para desarrollar las competencias. Independientemente de la competencia que se quiera trabajar, estas deberían ser activas, centradas en el estudiante, participativas, donde se les ofrezca a los alumnos múltiples situaciones contextualizadas, reales y variadas. Evidentemente, para adquirir las competencias el alumno tiene que experimentarlas en primera persona. Con todo ello, las instituciones educativas deberían ir ayudando en esta dirección y apuntar por el cambio metodológico activo y participativo en sus aulas.

En relación a la competencia emprendedora, tras el análisis bibliográfico realizado, las metodologías más empleadas para su fomento son muy variadas, desde el estudio de casos, hasta el aprendizaje basado en problemas, el aprendizaje basado en proyectos, las simulaciones y juegos, el aprendizaje cooperativo,... como se apuntaba a lo largo del artículo.

\section{Referencias}

Álvarez, P.R. (2016). Competencias genéricas en la enseñanza universitaria. De la tutoría formativa a la integración curricular. Málaga: Ediciones Aljibe.

Arasti, Z., Kiani, M. y Imanipour, N. (2012). A Study of Teaching Methods in Entrepreneurship Education for Graduate Students. Higher Education Studies, 2(1), 2-10.

Arruti, A. (2016). El desarrollo del perfil del teacherpreneur o profesor-emprendedor en el currículum del Grado de Educación Primaria: ¿un concepto de moda o una realidad? Contextos educativos, 19, 177-194.

Asociación Red GEM España (2016). Global Entrepreneurship Monitor: informe GEM España. Santander: Editorial de la Universidad de Cantabria. Recuperado de http://www.gem-spain.com/wp-content/uploads/2015/03/Informe-GEM-2015esafp.pdf

Bager, T. (2011). Entrepreneurship education and new venture creation: a comprehensive approach. En K. Hindle. y K. Klyver (Eds.), Handbook of research on new venture creation. UK: Edgar Elgar Publishing.

Balan, P. y Metcalfe, M. (2012). Identifying teaching methods that engage entrepreneurship students. Education + Training, 54(5), 368-384. 
Coduras, A, Levie, J., Kelley, D., Saemundsson, R. y Schott, T. (2010). Global Entrepreneurship Monitor Special Report: Una Perspectiva Global sobre la Educación y Formación Emprendedora. Global Entrepreneurship Research Association (GERA). Recuperado de

http://datateca.unad.edu.co/contenidos/256593/Evaluacion_final/Perspectiva_Globa I_Formacion_y_Educacion.pdf

Coloma, A. M., Jiménez, M. Á. y Sáez, A. M. (2007). Metodologías para desarrollar competencias y atender a la diversidad. Guía para el cambio metodológico y ejemplos desde infantil hasta la universidad. Madrid: PPC Editorial.

Comisión Europea (2008). Entrepreneurship in Higher Education, especially in non-business studies.

Comisión Europea (2016a). EntreComp: The Entrepreneurship Competence Framework. Recuperado http://publications.jrc.ec.europa.eu/repository/bitstream/JRC101581/lfna27939enn.p $\mathrm{df}$

Comisión Europea (2016b). Entrepreneurship Education at School in Europe. Eurydice Report. Luxembourg: Publications Office of the European Union. Recuperado de https://webgate.ec.europa.eu/fpfis/mwikis/eurydice/images/4/45/195EN.pdf

Damián, J. (2013). Sistematizando experiencias sobre educación en emprendimiento en escuelas de nivel primaria. Revista Mexicana de Investigación Educativa, 18, 159-190.

De la Fuente, J., Vera, M.M. y Cardelle-Elawar, M. (2012). Aportaciones de la Psicología de la Innovación y del Emprendimiento a la Educación en la Sociedad del Conocimiento. Electronic Journal of Research in Educational Psychology, 10(3), 941-966.

De Miguel, M. (Coord.) (2005). Modalidades de Enseñanza centradas en el desarrollo de Competencias: orientaciones para promover el cambio metodológico en el Espacio Europeo de Educación Superior. Proyecto EA2005-0118.

De Miguel, M. (Coord.) (2006). Metodologías de enseñanza y aprendizaje para el desarrollo de competencias. Orientaciones para el profesorado universitario ante el Espacio Europeo de Educación Superior. Madrid: Alianza Editorial.

Etzkowitz, H. y Leydesdorff, L. (1998). The endless transition: A "triple helix" of universityindustry-government relations. Minerva, 36(3), 203-08.

Fayolle, A., Gailly, B. y Lassas-Clerc, N. (2006). Assessing the impact of entrepreneurship education programmes: a new methodology. Journal of European Industrial Training, 30(9), 701-720.

Fernández, A. (2006). Metodologías activas para la formación de competencias. Educatio siglo XXI, 24, 35-56.

Fiet, J.O. (2001a). The Pedagogical Side of Entrepreneurship Theory. Journal of Business Venturing, 16, 101-117.

Fiet, J.O. (2001b). The theoretical side of teaching entrepreneurship. Journal of Business Venturing, 16, 1-24.

Garavan, T.N. y O'Cinneide, B. (1994). Entrepreneurship Education and Training Programmes: A Review and Evaluation - Part 1. Journal of European Industrial Training, 18(8), 3-12. 
García, R., Traver, J.A. y Candela, I. (2001). Aprendizaje cooperativo. Fundamentos, características y técnicas. Madrid: Editorial CCS.

Gibb, A. y Hannon, P. (2007). Towards the Entrepreneurial University. International Journal of Entrepreneurship Education, 4, 73-110.

González, J. y Wagenaar, R. (Eds.). (2003). Tuning Educational Structures in Europe. Informe Final. Fase Uno. Deusto: Universidad de Deusto.

Henry, C., Hill, F. y Leitch, C. (2005). Entrepreneurship education and training: can entrepreneurship be taught? Part 1. Education and Training, 47(2), 98-111.

Hindle, K. (2007). Teaching entrepreneurship at university: from the wrong building to the right philosophy. En Handbook of Research in Entrepreneurship Education, Volume 1.

Hoffmann, A., Fuglsang, T. y Vestergaard, L. (2012). Measuring Entrepreneurship Education. En Entrepreneurship determinants: culture and capabilities. European Commission. Luxembourg: Publications Office of the European Union. Recuperado de http://ec.europa.eu/eurostat/documents/3217494/5748437/KS-31-12-758-EN.PDF

Honig, B. (2004). Entrepreneurship Education: Toward a Model of Contingency-Based Business Planning. Academy of Management Learning and Education, 3(3), 258-273.

Hytti, U. y O'Gorman, C. (2004). What is "enterprise education"? An analysis of the objectives and methods of enterprise education programmes in four European countries. Education + Training, 46(1), 11-23.

Jiménez, G., Elías, R. y Silva, C. (2014). Innovación docente y su aplicación al EEES: Emprendimiento, TIC y Universidad. Historia y Comunicación Social, 19, 187-196.

Jiménez, R. (Coord.). (2015). Educación emprendedora. Programa TALOS para el desarrollo de la iniciativa emprendedora en Ciencias de la Educación. Barcelona: Editorial Octaedro.

Jones, B. y Iredale, N. (2010). Enterprise education as pedagogy. Education + Training, 52(1), 7-19.

Kirby, D.A. (2004). Entrepreneurship education: can business schools meet the challenge? Education + Training, 46(8), 510-519.

Koch, L.T. (2003). Theory and Practice of Entrepreneurship Education: A German View. International Journal of Entrepreneurship Education, 1(4), 633-660.

Kuratko, D. (2003). Entrepreneurship education: emerging trends and challenges for the 21st century. Coleman Foundation White Paper Series for the U.S. Association of Small Business \& Entrepreneurship.

Kuratko, D. (2005). The Emergence of Entrepreneurship Education: Development, Trends and Challenges. Entrepreneurship Theory and Practice, 29(5), 577-98.

Labrador, M. J. y Andreu, M. Á. (2008). Metodologías activas. Grupo de Innovación en metodologías activas (GIMA). Valencia: Editorial UPV.

Ley Orgánica 10/2002, de 23 de diciembre, de Calidad de la Educación. Boletín Oficial del Estado, 24 de diciembre de 2002, 307, pp. 45188-45220.

Ley Orgánica 2/2006, de 3 de mayo, de Educación. Boletín Oficial del Estado, 4 de mayo de 2006, 106, pp. 17158-17207.

Ley $14 / 2013$, de 27 de septiembre, de apoyo a los emprendedores y su internacionalización. Boletín Oficial del Estado, 28 de septiembre de 2013, 233, pp. 78787-78882. 
Ley Orgánica 8/2013, de 9 de diciembre, para la Mejora de la Calidad Educativa. Boletín Oficial del Estado, 10 de diciembre de 2013, 295, pp. 97858-97921.

Ministerio de Educación, Cultura y Deporte (2016). Eurydice España - REDIE. La educación para el emprendimiento en el sistema educativo español. Año 2015. Recuperado de https://sede.educacion.gob.es/publiventa/d/20842/19/1

Moust, J., Bouhuijs, P. y Schmidt, H. (2007). El aprendizaje basado en problemas: guía del estudiante. Cuenca: Ediciones de la Universidad de Castilla-La Mancha.

Samwel, E. (2010). Entrepreneurship education: a review of its objectives, teaching methods, and impact indicators. Education + Training, 52(1), 20-47.

Neck, H.M. y Greene, P.G. (2011). Entrepreneurship Education: Known Worlds and New Frontiers. Journal of Small Business Management, 49(1), 55-70.

Orden ECD/65/2015, de 21 de enero, por la que se describen las relaciones entre las competencias, los contenidos y los criterios de evaluación de la educación primaria, la educación secundaria obligatoria y el bachillerato. Boletín Oficial del Estado, 29 de enero de 2015, 25, pp. 6986-7003. Recuperado de https://www.boe.es/boe/dias/2015/01/29/pdfs/BOE-A-2015-738.pdf

Pellicer, C., Álvarez, B. y Torrejón, J.L. (2013). Aprender a emprender. Cómo educar el talento emprendedor. Grupo Planeta.

Powell, B.C. (2013). Dilemmas in entrepreneurship pedagogy. Journal of Entrepreneurship Education, 16, 99-112.

Resolución de 30 de agosto de 2013, de la Dirección General de Política Educativa Escolar, por la que se dispone la publicación de la Instrucción de 30 de agosto de 2013 de esta Dirección General por la que se establecen orientaciones pedagógicas y se determinan las actuaciones, dirigidas a fomentar la cultura emprendedora, que los centros sostenidos con fondos públicos en la Comunidad de Castilla y León que impartan educación primaria, educación secundaria obligatoria y bachillerato deberán realizar a partir del curso 2013-14. Boletín Oficial de Castilla y León, 11 de septiembre de 2013, 175, pp. 62082- 62096. Recuperado de http://www.educa.jcyl.es/es/resumenbocyl/resolucion-30-agosto-2013-direcciongeneral-politica-educ-1.ficheros/453353-BOCYL-D-11092013-18.pdf

Ripollés, M. (2011). Aprender a emprender en las universidades. Arbor Ciencia, Pensamiento y Cultura, 187, Extra 3, 83-88.

Rosales, C. (2013). Competencias específicas curriculares que ha de adquirir el estudiante del título de Grado de Maestro. Profesorado. Revista de currículum y formación del profesorado, 17(3), 73-90.

Ruiz, F.J. (2015). Competencia emprendedora. En L. Villardón-Gallego (Coord.). (2015). Competencias genéricas en educación superior. Metodologías específicas para su desarrollo, pp.103-140. Madrid: Narcea Ediciones.

Ruskovaara, E. y Pihkala, T. (2013). Teachers implementing entrepreneurship education: classroom practices. Education + Training, 55(2), 204 - 216.

Seikkula-Leino, J., Ruskovaara, E., Ikavalko, M., Mattila, J. y Rytkola, T. (2010). Promoting entrepreneurship education: the role of the teacher? Education + Training, 52(2), 117127. 
Sherman, P.S., Sebora, T. y Digman, L.A. (2008). Experiential entrepreneurship in the classroom: effects of teaching methods on entrepreneurial career choice intentions. Journal of Entrepreneurship Education, 11, 29-42.

Solomon, G.T., Duffy, S. y Tarabishy, A. (2002). The state of entrepreneurship education in the United States: A nationwide survey and analysis. International Journal of Entrepreneurship Education, 1(1), 65-86.

Solomon, G. (2007). An examination of entrepreneurship education in the United States. Journal of Small Business and Enterprise Development, 14(2), 168-182.

Unión Europea (2012). Entrepreneurship determinants: culture and capabilities. Recuperado de http://ec.europa.eu/eurostat/documents/3217494/5748437/KS-31-12-758-EN.PDF

Villa, A. y Poblete, M. (2007). Aprendizaje Basado en Competencias: una propuesta para la evaluación de las competencias genéricas. Bilbao: Ediciones Mensajero.

Volkmann, C. (2004). Entrepreneurship Studies-An Ascending Academic Discipline in the Twenty-First Century. Higher Education in Europe, 29(2), 177-185.

Winslow, E.K., Solomon, G.T. y Tarabishy, A. (1997, enero). Empirical Investigation into Entrepreneurship Education in the United States: Some Results of the 1997 National Survey of Entrepreneurial Education. XIII Conferencia Nacional Anual, Sailing the Entrepreneurial Wave into the 21st Century, San Diego.

Zabala, A. y Arnau, L. (2014). Métodos para la enseñanza de las competencias. Barcelona: Editorial Graó.

Zahra, S. y Welter, F. (2008). Entrepreneurship Education for Central, Eastern and Southeastern Europe. En J. Potter (Ed.). Entrepreneurship and Higher Education, OECD Publishing. 